\title{
Protocolo de assistência de enfermagem ao paciente em pré e pós-operatório de cirurgia bariátrica
}

\author{
Nursing care protocol to the patient before and after bariatric surgery \\ Protocolo de atención de enfermería al paciente antes y después de la cirugía bariátrica
}

\begin{abstract}
Lidiany Galdino Felix', Maria Júlia Guimarães Oliveira Soares", Maria Miriam Lima da Nóbrega'"
' Universidade Federal de Campina Grande, Centro de Ciências Biológicas e da Saúde, Curso de Enfermagem. Campina Grande-PB, Brasil.

"Universidade Federal da Paraíba, Centro de Ciências da Saúde, Departamento de Enfermagem Clínica, Programa de Pós-Graduação em Enfermagem, Grupo de Estudos e Pesquisas no Tratamento de Feridas. João Pessoa-PB, Brasil. I"I Universidade Federal da Paraíba, Departamento de Enfermagem de Saúde Pública e Psiquiatria, Programa de Pós-Graduação em Enfermagem, Grupo de Estudos e Pesquisa em Fundamentação da Assistência de Enfermagem, João Pessoa-PB, Brasil.
\end{abstract}

Submissão 05-11-2010 Aprovação: 04-01-2012

\section{RESUMO}

Estudo do tipo metodológico e de inovação tecnológica, que objetivou construir um protocolo de assistência de enfermagem para o paciente em pré e pós-operatório de cirurgia bariátrica. O modelo de assistência proposto fundamentou-se na Teoria Geral do Autocuidado de Orem e foi organizado a partir dos requisitos de autocuidado (universais, de desenvolvimento e de desvios de saúde). Para cada requisito, são apresentados os diagnósticos de enfermagem, os resultados esperados e as intervenções de enfermagem, identificados em pesquisa envolvendo 31 clientes portadores de obesidade mórbida, assistidos no Ambulatório de Gastroenterologia de um hospital universitário, no período de abril a junho de 2009. Considera-se o desenvolvimento do protocolo um importante recurso para instrumentalizar as orientações prestadas pelos enfermeiros ao paciente bariátrico, visando ao autocuidado, além de dar subsídios para sistematizar a assistência de enfermagem a ser prestada. Descritores: Enfermagem; Autocuidado; Cirurgia bariátrica; Protocolos de enfermagem.

\section{ABSTRACT}

This is a methodological study of innovative technology developed which aims to build up nursing care protocol to the patient in the pre and postoperative bariatric surgery. The proposed model of care was based on the General Theory Orem Self-Care and organized from the requirements of self-care (universal, developmental and health deviation). Within each requirement, it is presented nursing diagnoses, expected outcomes and nursing interventions that were identified in a survey conducted with 31 clients suffering from morbid obesity, assisted by the Gastroenterology Outpatient Clinic of a university hospital in the period from April to June 2009. The development of the protocol is considered an important resource both to organize the guidelines provided by nurses to the bariatric patient, aiming their self-care, and to provide subsidies to systematize nursing care to be provided Key words: Nursing; Self care; Bariatric surgery; Nursing Protocols.

\section{RESUMEN}

Estudio del tipo metodológico y de innovación tecnológica desarrollado con el objetivo de construir un protocolo de asistencia de enfermería al paciente en pre y pos-operatorio de cirugía bariátrica. El modelo de asistencia propuesto fue fundamentado en la Teoría General del Auto-cuidado de Orem y organizado a partir de los requisitos de auto-cuidado (universales, de desarrollo y de desvíos de la salud). Dentro de cada requisito, son presentados los diagnósticos de enfermería, los resultados esperados y las intervenciones de enfermería que fueron identificados en investigación realizada con 31 clientes portadores de obesidad morbosa, asistidos por el Dispensario de Gastroenterología de un hospital universitario en el período de abril a junio de 2009. Se considera el desarrollo del protocolo un importante recurso para instrumentalizar las orientaciones prestadas por los enfermeros al paciente bariátrico, visando su auto-cuidado además de fornecer subsidios para sistematizar la asistencia de enfermería a ser prestada.

Palabras clave: Enfermería; Auto-cuidado; Cirugía bariátrica; Protocolos de Enfermería.

Extraído de dissertação de Mestrado apresentada ao Programa de Pós-Graduação em Enfermagem, Centro de Ciências da Saúde, Universidade Federal da Paraíba, João Pessoa-PB, 2009. Trabalho agraciado com o segundo lugar no prêmio "Laís Neto dos Reis", $62^{\circ}$ Congresso Brasileiro de Enfermagem, Florianópolis-SC, 2010. 


\section{INTRODUÇÃO}

A obesidade é uma doença complexa, caracterizada pelo acúmulo excessivo de gordura no organismo, que está diretamente relacionada ao surgimento de doenças crônicas como: diabetes tipo II, hipertensão arterial, dislipidemias, coronariopatias, doenças articulares, cálculos biliares, apneia do sono e muitos tipos de câncer(1).

Devido aos riscos associados, a obesidade vem sendo considerada um grande problema de saúde pública, que afeta todas as faixas etárias e os grupos socioeconômicos( ${ }^{(2)}$, tanto em países desenvolvidos quanto em desenvolvimento, com consequências sociais e psicológicas graves e de proporções epidêmicas ${ }^{(3)}$.

Atualmente, a cirurgia bariátrica vem sendo o principal tratamento para a redução de peso e a melhora das comorbidades associadas à obesidade. No Brasil, o avanço das técnicas cirúrgicas e a popularização da cirurgia promoveram a expansão dos cuidados de enfermagem para essa clientela. Contudo, apesar da importância da temática e do interesse de vários países nesse assunto, a atuação da Enfermagem nessa área ainda está voltada para diretrizes médicas, haja vista o pouco número de publicações realizadas em nosso país, voltadas, especificamente, para a assistência de enfermagem prestada ao paciente submetido à cirurgia bariátrica ${ }^{(4-5)}$.

Como forma de aprimorar os conhecimentos de enfermagem na área e propor novas alternativas de assistência voltadas para o autocuidado do paciente e para o alcance do sucesso da cirurgia, propomos, neste estudo, construir um protocolo de assistência de enfermagem para o paciente em pré e pós-operatório de cirurgia bariátrica, fundamentado na Teoria Geral do Autocuidado de Orem ${ }^{(6)}$.

O uso do referencial teórico de Orem, neste trabalho, justifica-se pelo fato de a teoria enfatizar a importância do engajamento do cliente para o autocuidado, estimulando-o a participar, de forma ativa, da sua recuperação. Além de ser um dos modelos que pode direcionar as ações assistenciais do enfermeiro e responder às necessidades de portadores de doenças crônicas como a obesidade, facilita o planejamento da assistência de enfermagem para esses pacientes ${ }^{(7)}$.

\section{MÉTODO}

O estudo é do tipo metodológico e de tecnologia assistencial, visto que a construção de protocolos assistenciais em enfermagem está relacionada a uma proposta de inovação tecnológica. Embora se conceitue tecnologia em enfermagem como a aplicação sistemática de conhecimentos científicos para facilitar o processo de atendimento ao ser humano, constata-se o fato de que as técnicas com evidências científicas na Enfermagem valorizam a profissão ${ }^{(8)}$.

Considerando que a realização de estudos sobre o uso de materiais educativos, como, por exemplo, os manuais e protocolos assistenciais, no processo de recuperação pós-operatória, ainda são incipientes em nosso meio ${ }^{(9)}$, a construção desse protocolo pode ser entendida como um ponto de partida para outras pesquisas de natureza semelhante.
Para o alcance do objetivo proposto, o estudo foi desenvolvido no Ambulatório de Gastroenterologia de um Hospital Universitário, na cidade de João Pessoa - PB, organizado em quatro etapas: 1) identificação dos indicadores empíricos para a demanda terapêutica de autocuidado na literatura pertinente e estruturação do instrumento para a coleta de dados; 2 ) determinação dos déficits de autocuidado; 3 ) desenvolvimento dos sistemas de enfermagem para os déficits de autocuidado identificados; e 4) estruturação do protocolo de orientação para a assistência de enfermagem ao paciente em pré e pós-operatório de cirurgia bariátrica.

Para operacionalizar a pesquisa e atender à Resolução 196/96, o projeto foi encaminhado e aprovado pelo Comitê de Ética e Pesquisa do Hospital Universitário Lauro Wanderley/UFPB, sob o Parecer nº 003/2009.

\section{RESULTADOS E DISCUSSÃO}

\section{Identificação dos indicadores empíricos para a demanda terapêutica de autocuidado e estruturação do instrumen- to para a coleta de dados}

Dentro da hierarquia do conhecimento de Enfermagem, os indicadores empíricos são considerados os critérios e/ou as condições experimentais, que são usados para observar ou mensurar os conceitos de uma teoria ${ }^{(10-11)}$. Por se tratar de um estudo metodológico baseado na Teoria do Autocuidado de Orem, os indicadores empíricos subsidiaram a estruturação do instrumento para a coleta de dados e foram considerados como as manifestações observadas ou mensuradas dos requisitos de autocuidado afetados no cliente com obesidade mórbida.

Para identificar esses indicadores, foi realizada uma revisão sistemática de periódicos nacionais e internacionais disponíveis na Biblioteca Virtual de Saúde (BVS), indexadas nas bases de dados MEDILINE, LILACS e SciELO, com os descritores "cirurgia bariátrica", "enfermagem" e "autocuidado", além de edições impressas de livros e revistas sobre os cuidados de enfermagem com o paciente submetido à cirurgia bariátrica e de trabalhos que associem a Teoria do Autocuidado à assistência de enfermagem a essa clientela.

A revisão sistemática teve por objetivo identificar os indicadores empíricos para as Demandas Terapêuticas de Autocuidado e subsidiar a construção do instrumento para a coleta de dados dos requisitos de autocuidado (universais, de desenvolvimento e de desvios de saúde) nos pacientes no pré e pós-operatório de cirurgia bariátrica.

Para a escolha das publicações, foram considerados os seguintes critérios de inclusão: ser artigo bibliográfico ou de pesquisa, ter sido publicado no período de 1999 a 2008, estar escrito em português, inglês ou espanhol, ter disponibilizado acesso completo via on line.

A busca bibliográfica, segundo estratégia definida, resultou em 1.210 estudos, dos quais 1.142 foram encontrados na MEDLINE, 42, no LILACS, e 26, no SciELO. Inicialmente, procedeu-se à leitura dos títulos e resumos dos artigos, com a finalidade de identificar as referências que se adequassem aos critérios pré-estabelecidos. Dessa forma, foram descartados 
1.128 estudos, em decorrência das seguintes razões: conteúdo não relacionado com a questão da pesquisa; título incoerente com o objeto de estudo realizado e os que se encontravam indexados em mais de uma base de dados, o que resultou em um total de 82 estudos.

De posse dos 82 artigos selecionados, foi realizada uma leitura exploratória e analítica deles, com a finalidade de ordenar e sumarizar as informações contidas, dos quais foram excluídos 56. Com a leitura dos 26 artigos restantes, foi possível identificar os indicadores empíricos para os requisitos de autocuidado presentes na literatura e construir o instrumento para a coleta de dados dos requisitos para o autocuidado em pacientes no pré e pós-operatório de cirurgia bariátrica.

Destaca-se, ainda, que, entre os periódicos nacionais selecionados, apenas um artigo ${ }^{(5)}$ estava voltado especificamente para a assistência de enfermagem ao paciente submetido à cirurgia bariátrica e evidenciava a importância da participação do enfermeiro na equipe multidisciplinar que assiste o paciente obeso.

Após a realização da revisão sistemática da literatura e a identificação dos indicadores empíricos para a demanda de autocuidado, foi estruturado o formulário para a coleta de dados.

\section{Determinação dos déficits de autocuidado e desenvolvi- mento dos sistemas de enfermagem}

Para determinar os déficits de autocuidado, foi realizado um estudo exploratório e descritivo, com abordagem quantiqualitativa, com pacientes portadores de obesidade mórbida. Os dados foram coletados por meio de entrevistas realizadas no período de abril a junho de 2009, no Ambulatório de Gastroenterologia do hospital campo da pesquisa.

A amostra foi constituída por acessibilidade e composta por 31 pacientes portadores de obesidade mórbida, em condições físicas e emocionais de responder às perguntas e que se dispuseram a participar da pesquisa. Para selecionar os pacientes que participariam da pesquisa, foram adotados os seguintes critérios: ter idade maior de 18 anos, por serem legalmente capazes, de acordo com a Legislação Brasileira, e voluntariedade para participar da pesquisa. Os pacientes foram incluídos no estudo depois que assinaram o Termo de Consentimento Livre e Esclarecido. Dos 31 participantes da pesquisa, 25 (80,6\%) eram candidatos à realização da cirurgia bariátrica, e seis $(19,4 \%)$ estavam em pós-operatório - dois, em pós-operatório mediato, e quatro, em pós-operatório tardio.

Em relação ao Índice de Massa Corpórea (IMC) dos entrevistados, 93,5\% (29) dos pacientes apresentavam obesidade grau III (IMC > $\left.40 \mathrm{~kg} / \mathrm{m}^{2}\right) ; 3,2 \%$ (1), obesidade grau II (IMC entre 35 e $39,9 \mathrm{~kg} / \mathrm{m}^{2}$ ); e 3,2\% (1), obesidade grau I (IMC entre 30 e $34,9 \mathrm{~kg} / \mathrm{m}^{2}$ ). $77,4 \%$ dos participantes eram do gênero feminino, e 22,6\% do gênero masculino. O predomínio de mulheres na amostra decorre do fato de procurarem mais o tratamento para o controle da obesidade, já que não houve direcionamento por sexo na inclusão dos pacientes na amostra(3).

A abordagem do processo de enfermagem proposta por Orem $^{(6)}$ apresenta um método para determinar as deficiências de autocuidado e definir, posteriormente, os papéis da pessoa ou do enfermeiro que irá satisfazer às exigências de autocuidado. Compreende as fases de diagnóstico e prescrição, planejamento de um sistema de enfermagem e plano para a execução do atendimento, produção e execução dos sistemas de enfermagem ${ }^{(4)}$.

Nessa perspectiva, a partir das demandas de autocuidado apresentadas, foram identificados os déficits de autocuidado e elaborados os respectivos diagnósticos de enfermagem. Para a construção dos diagnósticos de enfermagem, utilizou-se a Taxonomia II da NANDA-I I ${ }^{(12)}$, por ser o sistema de classificação de diagnósticos de enfermagem mais divulgado e utilizado pela Enfermagem atualmente.

Diante dos resultados obtidos, os diagnósticos de enfermagem identificados para os déficits de autocuidado apresentados pelos pacientes foram: Distúrbio da Imagem Corporal (90,3\%); Conhecimento deficiente sobre complicações relacionadas à obesidade $(83,9 \%)$; Interação social prejudicada $(67,7 \%)$; Padrão respiratório ineficaz (58,1\%); Nutrição desequilibrada: maior do que as necessidades corporais (54,8\%); Padrão de sono perturbado (54,8\%); Constipação (54,5\%); Diarreia (45,5\%) e Estilo de vida sedentário (45,2\%).

Durante a realização das entrevistas, observou-se que, apesar da importância da cirurgia para o tratamento da obesidade e de todas as esperanças depositadas pelo paciente no procedimento cirúrgico, eles não tinham muitas informações a respeito dessa intervenção, tendo em vista que, entre os 25 candidatos à cirurgia bariátrica entrevistados, 40,0\% disseram que sabiam pouco ou quase nada a respeito da cirurgia, o que levou ao diagnóstico de enfermagem: conhecimento deficiente em relação à cirurgia bariátrica.

Entre os pacientes em pós-operatório, 33,3\% referiram não ter sido informados sobre nenhuma complicação pós-operatória relacionada à cirurgia. Observou-se também que a maioria dos esclarecimentos a respeito desse procedimento cirúrgico, principalmente entre os pacientes que estavam em pós-operatório, veio através de pesquisas na Internet, da observação e relatos de experiências de amigos e familiares que haviam realizado a cirurgia anteriormente.

Constitui direito do paciente a precisa informação sobre os detalhes do procedimento cirúrgico a que irá se submeter, com a análise profissional de seus fatores de risco e complicações possíveis de ocorrer, bem como, de total acesso ao cirurgião e sua equipe multidisciplinar durante todas as fases de seu tratamento ${ }^{(13)}$, para que os pacientes não recebam informações conflitantes ou pouco precisas a respeito dessa intervenção cirúrgica.

Diante da predominância dos diagnósticos de enfermagem relacionados ao conhecimento deficiente sobre a cirurgia bariátrica e suas complicações, tem-se a convicção de que a educação e o preparo pré-operatório são importantes para a recuperação do paciente e para o alcance do sucesso da cirurgia.

A partir da identificação dos diagnósticos de enfermagem, foi realizado o planejamento da assistência, que envolveu a determinação de metas, objetivos, método de ajuda e do tipo de sistema de enfermagem a ser estabelecido nas intervenções de enfermagem, conforme preconiza o processo de enfermagem proposto por Orem ${ }^{(6)}$. 
Quase todos os sistemas de enfermagem determinados foram baseados em ações de enfermagem de apoio e educação, o que mostra a coerência na decisão pela Teoria do Autocuidado para nortear o processo de enfermagem em portadores de obesidade mórbida, que precisam de orientação, principalmente pela dificuldade de aderir ao tratamento e pelas comorbidades que a doença produz.

Todos os elementos identificados na coleta de dados e na análise dos discursos subsidiaram a construção do protocolo para orientar a assistência de enfermagem, que tem como objetivo sistematizá-la, visando ao autocuidado do paciente bariátrico, para ajuda-lo a conhecer e adotar comportamentos saudáveis para o alcance da finalidade maior da cirurgia - a redução do peso.

\section{Estruturação do Protocolo}

O protocolo técnico é um instrumento normativo do processo de intervenção técnica e social, que orienta os profissionais na realização de suas funções, e tem como base conhecimentos científicos e práticos do cotidiano do trabalho em saúde, de acordo com cada realidade ${ }^{(14)}$. As recomendações contidas em um protocolo devem advir de resultados de pesquisa, em que se devem empregar estratégias que facilitem a passagem dos resultados para a prática clínica ${ }^{(15)}$.

O protocolo desenvolvido neste estudo tem por objetivo instrumentalizar as orientações fornecidas pelos enfermeiros que atuam na assistência aos pacientes com obesidade mórbida, durante o pré e o pós-operatório de cirurgia bariátrica, para que possam realizar o seu autocuidado de forma segura e eficaz, além de fornecer subsídios para sistematizar a assistência de enfermagem a ser prestada.

O modelo de assistência proposto, apresentado no Quadro 1 , foi organizado com base nos requisitos de autocuidado universais, de desenvolvimento e de desvios de saúde, propostos pelo referencial teórico utilizado. Dentro de cada requisito, são apresentados os diagnósticos de enfermagem, os resultados esperados e as intervenções de enfermagem identificadas na pesquisa.

Esse material está sujeito a validação clínica e a avaliações e reformulações, conforme os avanços tecnológicos e científicos das técnicas cirúrgicas disponíveis atualmente.

Protocolo de orientação para a assistência de enfermagem ao paciente em pré e pós-operatório de cirurgia bariátrica

\begin{tabular}{|c|c|}
\hline \multicolumn{2}{|r|}{ REQUISITO DE AUTOCUIDADO: UNIVERSAIS } \\
\hline \multicolumn{2}{|r|}{ OXIGENAÇÃO } \\
\hline $\begin{array}{l}\text { DIAGNÓSTICO } \\
\text { DE ENFERMAGEM }\end{array}$ & Padrão respiratório ineficaz \\
\hline $\begin{array}{l}\text { RESULTADOS } \\
\text { ESPERADOS }\end{array}$ & Manterá um padrão respiratório eficaz/normal. \\
\hline $\begin{array}{l}\text { INTERVENÇÕES } \\
\text { DE ENFERMAGEM }\end{array}$ & $\begin{array}{l}\text { Pré-operatório } \\
\text { - Explicar a importância dos movimentos respiratórios e de tossir para a prevenção de complicações; } \\
\text { - Incentivar o exercício da tosse, para aumentar o volume inspiratório e expiratório; } \\
\text { - Mostrar ao paciente a importância de seu posicionamento no leito com a cabeceira elevada de } 45 \text { a } 75 \text { graus; } \\
\text { - Explicar que, quando estiver sentado, deverá posicionar os braços com almofadas na lateral, deixando o pulmão mais } \\
\text { livre para a sua expansão e as pernas estendidas. } \\
\text { Pós-operatório imediato } \\
\text { - Manter controle rigoroso dos sinais vitais e observar nível de consciência, saturação de oxigênio e perfusão periférica } \\
\text { dos membros; } \\
\text { - Avaliar o padrão respiratório e administrar oxigênio suplementar, quando prescrito; } \\
\text { - Realizar ausculta pulmonar, a fim de verificar as condições dos rúdos respiratórios; } \\
\text { - Manter a cabeceira do leito elevada de } 45 \text { a } 75 \text { graus; } \\
\text { - Incentivar a mudança de decúbito; } \\
\text { - Estimular a manutenção dos exercícios respiratórios (tosse e respiração profunda), promovendo a expansibilidade e } \\
\text { mobilidade das secreçôes; } \\
\text { - Ensinar ao paciente como imobilizar a incisão durante a tosse; } \\
\text { - Realizar medidas preventivas para aspiração (vômitos), caso seja necessário; } \\
\text { - Estimular a deambulação precoce; } \\
\text { - Mostrar ao paciente a importância de seu posicionamento no leito com a cabeceira elevada de } 45 \text { a } 75 \text { graus. } \\
\text { Pós-operatório tardio } \\
\text { - Orientar o paciente a monitorar sinais de desconforto respiratório, febre e acúmulo de secreções. }\end{array}$ \\
\hline \multicolumn{2}{|r|}{ HIDRATAÇÃO } \\
\hline $\begin{array}{l}\text { DIAGNÓSTICO } \\
\text { DE ENFERMAGEM }\end{array}$ & Risco para volume de líquidos deficiente \\
\hline $\begin{array}{l}\text { RESULTADOS } \\
\text { ESPERADOS }\end{array}$ & Aumentará a ingestão de líquidos diariamente. \\
\hline
\end{tabular}




\begin{tabular}{|c|c|}
\hline $\begin{array}{l}\text { INTERVENÇÕES } \\
\text { DE ENFERMAGEM }\end{array}$ & $\begin{array}{l}\text { Pré-operatório } \\
\text { - Orientar o paciente sobre a importância da manutenção da hidratação corporal. } \\
\text { Pós-operatório imediato: } \\
\text { - Realizar controle rigoroso do gotejamento das soluções parenterais; } \\
\text { - Monitorar balanço hídrico. } \\
\text { Pós-operatório tardio } \\
\text { - Reforçar as orientações pré-operatórias; } \\
\text { - Enfatizar a importância de não ingerir líquidos } 15 \text { minutos antes de cada refeição e até } 90 \text { minutos depois, para evitar } \\
\text { desconforto gástrico, pois beber e comer juntos aumenta a distensão gástrica, o que resulta em sensação de estufamento } \\
\text { e até vômitos; } \\
\text { - Reforçar que a ingestão de líquidos que, além de evitar a desidratação, auxilia na perda de peso, deve ser feita gradativa- } \\
\text { mente de acordo com a capacidade gástrica. }\end{array}$ \\
\hline \multicolumn{2}{|r|}{ ALIMENTAÇÃO } \\
\hline $\begin{array}{l}\text { DIAGNÓSTICO } \\
\text { DE ENFERMAGEM }\end{array}$ & Nutrição desequilibrada: maior do que as necessidades corporais \\
\hline $\begin{array}{l}\text { RESULTADOS } \\
\text { ESPERADOS }\end{array}$ & $\begin{array}{l}\text { Demonstrará alterações apropriadas no estilo de vida para o alcance de uma nutrição necessária ao atendimento das suas } \\
\text { necessidades metabólicas }\end{array}$ \\
\hline $\begin{array}{l}\text { INTERVENÇÕES } \\
\text { DE ENFERMAGEM }\end{array}$ & $\begin{array}{l}\text { Pré-operatório } \\
\text { - Explicar a importância da quantidade e da frequência da dieta prescrita, já estabelecida pela nutrição hospitalar; } \\
\text { - Monitorar a perda de peso; } \\
\text { - Orientar sobre o jejum. A American Society of Anesthesiologist (ASA) recomenda um período de jejum de seis horas ou } \\
\text { mais, com alimentos leves e líquidos sem resíduos por duas ou quatro horas antes do procedimento cirúrgico, e preconiza } \\
\text { o jejum de oito horas após uma refeição composta por alimentos gordurosos, frutas ou carnes. } \\
\text { Pós-operatório imediato } \\
\text { - Verificar a introdução da dieta e sua aceitação pelo paciente; } \\
\text { - Atentar para qualquer desconforto ou intercorrências que houver com a introdução da dieta, a presença de náuseas, } \\
\text { vômitos ou diarreias após a dieta, prestando os cuidados necessários ao paciente. } \\
\text { Pós-operatório tardio } \\
\text { - Reforçar as orientações nutricionais; } \\
\text { - Estimular a adesão e a obediência às recomendações dietéticas; } \\
\text { - Orientar a ingerir lentamente os alimentos, mastigá-los por completo e não ingerir líquido com as refeições. }\end{array}$ \\
\hline \multicolumn{2}{|r|}{ ELIMINAÇÃO } \\
\hline \multirow{2}{*}{$\begin{array}{l}\text { DIAGNÓSTICOS } \\
\text { DE ENFERMAGEM }\end{array}$} & Diarreia \\
\hline & Constipação \\
\hline $\begin{array}{l}\text { RESULTADOS } \\
\text { ESPERADOS }\end{array}$ & $\begin{array}{l}\text { - Restabelecerá e manterá o padrão normal de funcionamento intestinal; } \\
\text { - Demonstrará comportamento apropriado para ajudar a eliminar os fatores causadores. }\end{array}$ \\
\hline $\begin{array}{l}\text { INTERVENÇÕES } \\
\text { DE ENFERMAGEM }\end{array}$ & $\begin{array}{l}\text { Pré-operatório } \\
\text { - Investigar as queixas de diarreia, dor ao evacuar ou urinar; } \\
\text { - Avaliar quanto à presença de dor durante a micção; } \\
\text { - Avaliar os hábitos intestinais do paciente; } \\
\text { - Investigar os fatores que geralmente estimulam a atividade intestinal e as interferências existentes; } \\
\text { - Rever a ingestão dietética diária; } \\
\text { - Determinar a ingestão de líquidos; } \\
\text { - Avaliar a utilização de medicamentos; avaliar as interações ou efeitos colaterais desses medicamentos sobre o padrão } \\
\text { intestinal do paciente; } \\
\text { - Examinar a região perianal para detectar hemorroidas, lesões cutâneas ou outras anormalidades. } \\
\text { Pós-operatório imediato e tardio } \\
\text { - Avaliar débito urinário no sistema de drenagem fechada ou o desejo do paciente e a distensão vesical; } \\
\text { - Auscultar o abdome para determinar a presença e a localização dos ruídos hidroaéreos; } \\
\text { - Monitorizar presença de íleo paralítico (os ruídos hidroaéreos deverão retornar em média de } 6 \text { a } 12 \text { h após a cirurgia), } \\
\text { flatulência, distensão abdominal; } \\
\text { - Orientar o paciente a comunicar desconforto em região suprapúbica; } \\
\text { - Registrar a coloração, o odor, a consistência, a quantidade e a frequência das evacuações; } \\
\text { - Verificar presença, aspecto, características e frequência de eliminações intestinais; } \\
\text { - Observar e anotar eliminações e outras perdas como: diurese, vômitos, débitos de drenos, ingestão hídrica; } \\
\text { - Medir diariamente o débito do dreno de Blake; } \\
\text { - Comunicar à equipe médica para tomar as condutas necessárias, como a necessidade de sondagem vesical de alívio. }\end{array}$ \\
\hline \multicolumn{2}{|r|}{ ATIVIDADE } \\
\hline $\begin{array}{l}\text { DIAGNÓSTICO } \\
\text { DE ENFERMAGEM }\end{array}$ & Estilo de vida sedentário \\
\hline
\end{tabular}




\begin{tabular}{|c|c|}
\hline $\begin{array}{l}\text { RESULTADOS } \\
\text { ESPERADOS }\end{array}$ & - Realizará atividades físicas, para o seu bem-estar geral, conforme suas limitações. \\
\hline $\begin{array}{l}\text { INTERVENÇÕES } \\
\text { DE ENFERMAGEM }\end{array}$ & $\begin{array}{l}\text { Pré-operatório } \\
\text { - Avaliar o nível de desenvolvimento, as habilidades motoras, a postura e a marcha do paciente; } \\
\text { - Avaliar o padrão de atividade física do paciente; } \\
\text { - Identificar as condições que possam concorrer para o sedentarismo; } \\
\text { - Estimular a prática de atividade física dentro dos limites de tolerância; } \\
\text { - Orientar o paciente para a prática de atividade física regular, combinada com a alimentação saudável, para promoção } \\
\text { e redução no peso corporal maior que apenas a alimentação de forma isolada, além de aumentar a perda de gordura, } \\
\text { preservar a massa magra e diminuir o depósito de gordura visceral. } \\
\text { Pós-operatório imediato } \\
\text { - Estimular a deambulação logo que possível; } \\
\text { - Estimular a movimentação passiva dos pés, através da flexão e extensão, para aumentar o retorno venoso e prevenir a } \\
\text { formação de coágulos nos membros inferiores ou pelve que podem suceder à cirurgia da obesidade; } \\
\text { - Orientar o paciente que, durante o período pós-operatório, a deambulação precoce e o posicionamento correto do pa- } \\
\text { ciente no leito são muito importantes na prevenção de complicações pulmonares e de trombose venosa profunda; } \\
\text { - Atentar para sinais de hipotensão ortostática (diminuição da pressão arterial, fraqueza, tontura e desmaio) quando o } \\
\text { paciente levantar. } \\
\text { Pós-operatório tardio } \\
\text { - Reforçar que a atividade física é muito importante para os pacientes submetidos à cirurgia bariátrica, pois a perda de } \\
\text { peso será mais rápida; } \\
\text { - Orientar o paciente que evite esforços físicos excessivos nos primeiros } 90 \text { dias. Caminhadas leves, de curta distância (pela } \\
\text { manhã, próximo ao almoço e à tarde), poderão ser feitas conforme a resistência, progredindo lentamente, aumentando } \\
01 \text { minuto por dia. }\end{array}$ \\
\hline & SONO E REPOUSO \\
\hline $\begin{array}{l}\text { DIAGNÓSTICO } \\
\text { DE ENFERMAGEM }\end{array}$ & Padrão de sono perturbado \\
\hline $\begin{array}{l}\text { RESULTADOS } \\
\text { ESPERADOS }\end{array}$ & $\begin{array}{l}\text { - Demonstrará melhora do padrão de sono/repouso; } \\
\text { - Identificará as intervenções apropriadas para promover o sono. }\end{array}$ \\
\hline $\begin{array}{l}\text { INTERVENÇÕES } \\
\text { DE ENFERMAGEM }\end{array}$ & $\begin{array}{l}\text { Pré-operatório } \\
\text { - Avaliar as queixas referentes à qualidade de sono; } \\
\text { - Avaliar o padrão de sono do paciente; } \\
\text { - Identificar as circunstâncias que interrompem o sono e a frequência com que ocorrem; } \\
\text { - Estimular o paciente a dormir com a cabeceira elevada, como forma de evitar o laringoespasmo e a obstrução das vias } \\
\text { aéreas superiores; } \\
\text { - Orientar a manter o ambiente calmo, tranquilo e com luzes apagadas e sem ruído durante a noite; } \\
\text { - Manter um horário regular para acordar, dormir e descansar; } \\
\text { - Orientar o paciente a limitar o tempo de sono durante o dia e evitar alimentos e bebidas contendo cafeína antes de dor- } \\
\text { mir, ler algo agradável e relaxante, não assistir à televisão na cama. } \\
\text { Pós-operatório imediato } \\
\text { - Estimular o paciente a dormir com a cabeceira elevada, como forma de evitar o laringoespasmo e a obstrução das vias } \\
\text { aéreas superiores; } \\
\text { - Explicar a necessidade das interrupções geradas pela monitoração dos sinais vitais e/ou outros cuidados; } \\
\text { - Planejar a assistência de modo a assegurar períodos ininterruptos de sono durante a noite; } \\
\text { - Manter o ambiente calmo, tranquilo e com luzes apagadas e sem ruído. } \\
\text { Pós-operatório tardio } \\
\text { - Explicar a importância das estratégias para manter o sono eficaz. }\end{array}$ \\
\hline \multicolumn{2}{|r|}{ SOLIDÃO E INTERAÇÃO SOCIAL } \\
\hline $\begin{array}{l}\text { DIAGNÓSTICO } \\
\text { DE ENFERMAGEM }\end{array}$ & Interação social prejudicada \\
\hline $\begin{array}{l}\text { RESULTADOS } \\
\text { ESPERADOS }\end{array}$ & $\begin{array}{l}\text { - Identificará estratégias para a sua socialização. } \\
\text { - Reconhecerá os fatores que causam ou as dificuldades nas interações sociais. } \\
\text { - Relatará o desejo de realizar mudanças positivas nos comportamentos sociais. }\end{array}$ \\
\hline $\begin{array}{l}\text { INTERVENÇÕES } \\
\text { DE ENFERMAGEM }\end{array}$ & $\begin{array}{l}\text { Pré-operatório } \\
\text { - Rever a história social com o cliente/família e identificar os fatores que levaram às alterações no comportamento social; } \\
\text { - Determinar os padrões familiares de relacionamento e os comportamentos sociais; } \\
\text { - Explicar ao paciente que a obesidade é uma doença e que tem tratamento; } \\
\text { - Avaliar a participação do paciente em atividades de lazer; } \\
\text { - Encorajar o paciente ao convívio social com os amigos e familiares e estimular sua participação em atividades que lhe } \\
\text { deem prazer; } \\
\text { - Orientar que a cirurgia bariátrica não é uma cirurgia milagrosa, mas serve como alternativa para obesos mórbidos, lev- } \\
\text { ando a uma melhora na sua qualidade de vida. } \\
\text { Pós-operatório tardio } \\
\text { - Motivar e estimular a participação do paciente nas atividades de autocuidado; } \\
\text { - Estimular o paciente a participar de atividades de lazer logo que possível. }\end{array}$ \\
\hline
\end{tabular}




\begin{tabular}{|c|c|}
\hline \multicolumn{2}{|r|}{ PREVENÇÃO DE RISCOS À VIDA E AO BEM ESTAR } \\
\hline $\begin{array}{l}\text { DIAGNÓSTICO } \\
\text { DE ENFERMAGEM }\end{array}$ & Conhecimento deficiente sobre as complicações relacionadas à obesidade \\
\hline $\begin{array}{l}\text { RESULTADOS } \\
\text { ESPERADOS }\end{array}$ & $\begin{array}{l}\text { - Verbalizará a intenção de praticar comportamentos saudáveis desejáveis para o controle da obesidade e prevenção de } \\
\text { suas complicações. }\end{array}$ \\
\hline $\begin{array}{l}\text { INTERVENÇÕES } \\
\text { DE ENFERMAGEM }\end{array}$ & 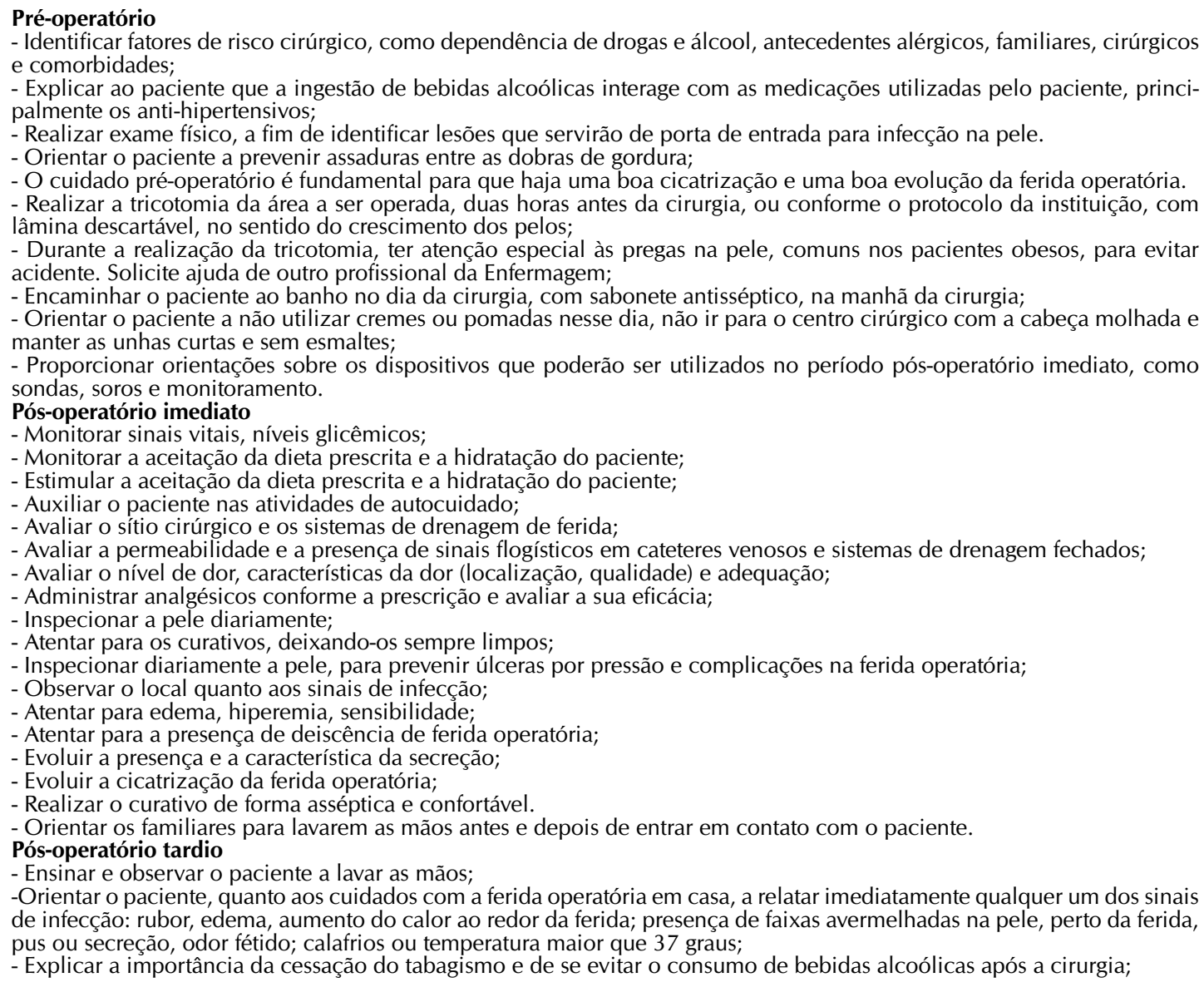 \\
\hline \multicolumn{2}{|r|}{ PROMOÇÃO DA SAÚDE } \\
\hline $\begin{array}{l}\text { DIAGNÓSTICO } \\
\text { DE ENFERMAGEM }\end{array}$ & Manutenção ineficaz da saúde \\
\hline $\begin{array}{l}\text { RESULTADOS } \\
\text { ESPERADOS }\end{array}$ & $\begin{array}{l}\text { - Demonstrará interesse em buscar ajuda para manter a saúde; } \\
\text { - Adotará comportamento para manter a saúde física e mental. }\end{array}$ \\
\hline $\begin{array}{l}\text { INTERVENÇÕES } \\
\text { DE ENFERMAGEM }\end{array}$ & $\begin{array}{l}\text { Pré-operatório } \\
\text { - Identificar os fatores causadores e relacionados que impedem o controle eficiente dos cuidados com a saúde do paciente; } \\
\text { - Fornecer informações relevantes sobre a importância do controle da pressão arterial e de níveis glicêmicos para o trata- } \\
\text { mento da obesidade; } \\
\text { - Explicar as etapas pelas quais ele irá passar antes e depois do procedimento cirúrgico; } \\
\text { - Enfatizar a importância do autocuidado e do engajamento do cliente nas recomendações nutricionais e de saúde, como } \\
\text { também a importância da cessação do fumo dois meses antes da cirurgia. } \\
\text {-Estimular a participação ativa do paciente e da família em todas as orientações pré-operatórias; } \\
\text { - Orientar o paciente que, para alcançar o sucesso da cirurgia, é preciso fazer mudanças de comportamento, dentre elas, } \\
\text { a reeducação alimentar e a prática regular de atividade física. } \\
\text { Pós-operatório imediato } \\
\text { - Estimular o paciente nas atividades de autocuidado, orientando-o sobre as finalidades dos procedimentos e as interven- } \\
\text { ções de enfermagem realizadas. } \\
\text { Pós-operatório tardio } \\
\text { - Orientar o paciente sobre a importância do monitoramento do controle do peso e do acompanhamento pós-operatório; } \\
\text { - Orientar sobre a importância do disciplinamento do paciente em relação às medicações e ao monitoramento de sua } \\
\text { saúde. } \\
\text { - Estimular a participação ativa do paciente e da família em todos os cuidados prestados. }\end{array}$ \\
\hline
\end{tabular}


REQUISITO DE AUTOCUIDADO DE DESENVOLVIMENTO

\begin{tabular}{|c|c|}
\hline $\begin{array}{l}\text { DIAGNÓSTICO } \\
\text { DE ENFERMAGEM }\end{array}$ & Distúrbio da imagem corporal \\
\hline $\begin{array}{l}\text { RESULTADOS } \\
\text { ESPERADOS }\end{array}$ & $\begin{array}{l}\text { - Demonstrará aceitação da sua condição e entendimento das mudanças no corpo após a cirurgia; } \\
\text { - Participará efetivamente das ações de autocuidado para a redução de peso. }\end{array}$ \\
\hline $\begin{array}{l}\text { INTERVENÇÕES } \\
\text { DE ENFERMAGEM }\end{array}$ & $\begin{array}{l}\text { Pré-operatório } \\
\text { - Estimular o paciente na prática de atividade física e reeducação alimentar para a efetiva perda de peso necessária antes } \\
\text { da realização da cirurgia. } \\
\text { Pós-operatório imediato } \\
\text { - Estimular a participação do pacientes nos cuidados prestados; } \\
\text { - Avaliar o engajamento do paciente e a participação da família nas atividades de autocuidado. } \\
\text { Pós-operatório tardio } \\
\text { - Avaliar a adaptação e a percepção do paciente às mudanças ocasionadas pela cirurgia. } \\
\text { - Estimular a participação do paciente em grupos de apoio para acompanhamento do processo de perda e de readaptação } \\
\text { às mudanças ocasionadas pela cirurgia. }\end{array}$ \\
\hline \multicolumn{2}{|r|}{ REQUISITO DE AUTOCUIDADO NOS DESVIOS DE SAÚDE } \\
\hline $\begin{array}{l}\text { DIAGNÓSTICO } \\
\text { DE ENFERMAGEM }\end{array}$ & Conhecimento deficiente com relação à cirurgia bariátrica \\
\hline $\begin{array}{l}\text { RESULTADOS } \\
\text { ESPERADOS }\end{array}$ & - Apresentará conhecimento sobre os aspectos relacionados à cirurgia bariátrica e suas complicações \\
\hline $\begin{array}{l}\text { INTERVENÇÕES } \\
\text { DE ENFERMAGEM }\end{array}$ & $\begin{array}{l}\text { Pré-operatório } \\
\text { Esclarecer ao paciente que: } \\
\text { - A obesidade é uma doença metabólica de origem multifatorial, que envolve fatores genéticos, endócrinos e ambientais; } \\
\text { - A obesidade mórbida é uma doença e tem tratamento; } \\
\text { - Os benefícios da cirurgia para a melhoria na qualidade de vida e o controle das doenças associadas à obesidade mórbida } \\
\text { e as etapas a serem percorridas, até realização de plásticas reparadoras no pós-operatóio; } \\
\text { - A indicação da cirurgia depende de alguns critérios de seleção de pacientes, os quais foram estabelecidos pela Federação } \\
\text { Internacional para a Cirurgia Bariátrica e são os seguintes: IMC maior que } 40 \mathrm{~kg} / \mathrm{m}^{2} \text { ou }>35 \mathrm{~kg} / \mathrm{m}^{2} \text { associado a comorbi- } \\
\text { dades de difícil manejo clínico, presença de obesidade por, no mínimo, cinco anos, fracasso no tratamento conservador, } \\
\text { ausência de história de alcoolismo ou problemas psiquiátricos graves, idade entre } 18 \text { e } 55 \text { anos e risco cirúrgico aceitável; } \\
\text { - Antes da realização da cirurgia, todo paciente deve ser submetido a uma avaliação minuciosa pré-operatória com exames } \\
\text { laboratoriais e de imagens, para afastar doenças que impossibilitem o ato cirúrgico ou que devam ser corrigidas antes } \\
\text { desse procedimento; } \\
\text { - Além disso, o paciente deverá fazer um acompanhamento com vários profissionais de saúde: endocrinologista, cirurgião, } \\
\text { enfermeiro, psicólogo e nutricionista, para auxiliar a adotar um novo estilo de vida, no engajamento para a reeducação } \\
\text { alimentar e perda de peso antes da cirurgia. } \\
\text { - Orientar o paciente sobre as principais complicações pós-operatória e sobre a importância do autocuidado para o suc- } \\
\text { esso da cirurgia. } \\
\text { Pós-operatório imediato } \\
\text { - Orientar o paciente sobre os procedimentos e as finalidades dos procedimentos e das intervenções de enfermagem } \\
\text { realizadas durante a internação. } \\
\text { - Auxiliar o paciente nas atividades de autocuidado; } \\
\text { - Estimular o paciente a participar das atividades de autocuidado; } \\
\text { Pós-operatório tardio } \\
\text { - Avaliar a adaptação e a percepção do paciente às mudanças ocasionadas pela cirurgia. }\end{array}$ \\
\hline
\end{tabular}

\section{Quadro 1 - Protocolo de assistência de enfermagem para o paciente em pré e pós-operatório de cirurgia bariátrica, fundamentado na Teoria de Autocuidado de Orem. João Pessoa, 2009.}

\section{CONCLUSÕES}

Considera-se o desenvolvimento do Protocolo proposto neste trabalho um recurso essencial para guiar o enfermeiro no planejamento da assistência, desde a fase ambulatorial até a internação e a alta hospitalar do paciente bariátrico. Além de ser um instrumento para a educação do paciente, visando ao seu autocuidado e à adoção de comportamentos saudáveis para sua redução do peso, pode ser de extrema utilidade na sua recuperação e no seu retorno às atividades cotidianas.
Deve ser enfatizado, ainda, que o processo de construção do Protocolo de Assistência de Enfermagem não finaliza neste estudo e que será um elemento para o desenvolvimento de novas pesquisas, pois esse instrumento deve ser submetido à validação clínica, com o objetivo de testar, em um grande número de clientes, sua viabilidade e operacionalidade. Essa validação será realizada em estudos posteriores, de forma a estimular e a melhorar a produção de conhecimentos e de pesquisas referentes à assistência de enfermagem nessa área. 


\section{REFERÊNCIAS}

1. Association of Perioperative Registred Nurses (AORN). Bariatric Surgery Guideline. AOURN 2004; 79(5):1026-52.

2. Lopes PCS, Prado SRLA, Colombo P. Fatores de risco associados à obesidade e sobrepeso em crianças em idade escolar. Rev Bras Enferm 2010 ;63(1):73-8.

3. Lima LP, Sampaio HAC. Caracterização socioeconômica, antropométrica e alimentar de obesos graves. Ciênc Saúde Coletiva. 2007;12(4):1011-20.

4. Felix LG, Nóbrega MML, Soares MJGO. Nursing process fundament in the nursing theory of Orem's self-care to a patient submitted to bariatric surgery. Rev Enferm UFPE On Line. 2009;3(4):26-34.

5. Negrão RJS, Bianchi ERF. A atuação do enfermeiro na assistência prestada ao paciente submetido à cirurgia bariátrica. Prát Hosp 2006;8(44):145-8.

6. Orem DE, Taylor S. Nursing: concepts of practice. 6th ed. New York: Mosby-Year Book; 2001.

7. Felix LG, Nóbrega MML, Fontes WD, Soares MJGO. Analysis from Theory of the Orem Self Care according to Fawcett criteria. Rev Enferm UFPE On Line. 2009;3(2):173-8.

8. Studart RMB, Melo EM, Lopes MVO, Barbosa IV, CarvaIho ZMF. Tecnologia de Enfermagem na prevenção da úlcera por pressão em pessoas com lesão medular. Rev Bras Enferm 2011;64(3):494-500.

9. Oliveira MS, Fernandes MSO, Sawada NO. Manual educativo para o autocuidado da mulher mastectomizada: um estudo de validação. Texto \& Contexto Enferm 2008;17(1):115-23.

10. Fawcett J. Criteria for evaluation of theory. Nurs Sci $Q$ 2005;18(2):131-5.

11. Souza APMA, Soares MJGO, Nóbrega MML. Indicadores empíricos para a estruturação de um instrumento de coleta de dados em unidade cirúrgica. Rev Eletrônica Enferm [periódico na internet]. 2009 [acesso em $13 \mathrm{dez}$ 2011];11(3).Disponível em: http://www.fen.ufg.br/revista/v11/n3/v11n3a06.htm.

12. North American Nursing Diagnosis Association - NANDA International. Diagnósticos de Enfermagem da NANDA: Definições e Classificação 2009-2011. Porto Alegre: Artmed; 2010.

13. Ferraz EM, Martins Filho EDM. Cirurgia bariátrica: indicação e importância do trabalho multidisciplinar. Einstein 2006;4(1):S71-2.

14. Conselho Regional de Enfermagem. Câmara Técnica da Atenção Básica [homepage na internet]. Apresentação de protocolos assistenciais. [acesso em $01 \mathrm{dez}$ 2009]. Disponível em: http:// www.coren-mg.gov.br/.../Apresentacao_Protocolos_Assistenciais_Rosana_Paes.pdf.

15. Borges EL, Saar SRC, Magalhães MBB, Gomes FSL, Lima VLAN. Feridas como tratar. 2th ed. Belo Horizonte: Coopemed; 2008. 